\title{
SLIT: Die Peakdosis ist entscheidend
}

- Für Patienten steht bei einer spezifischen Immuntherapie (SIT) die lang anhaltende Wirkung im Vordergrund. Das gaben drei Viertel der Teilnehmer einer bundesweiten Telefonumfrage im Jahr 2005 an. Knapp die Hälfte der Befragten wünschten sich daneben besonders eine rasche Wirksamkeit und über $41 \%$ wenig Nebenwirkungen, berichtete PD Dr. Randolf Brehler, Altenberge, anlässlich des 4 . Gemeinsamen Allergie-Kongresses. Für die Wirksamkeit der SIT ist das Erreichen einer hohen Peakdosis mit einer Erhaltungsdosis zwischen 5 und $20 \mu \mathrm{g}$ Major-Allergen pro Injektion von Bedeutung. Nur eine hohe Allergendosis induziere Toleranz, betonte Brehler.

Eine Quick-Aufdosierung mit Depiquick ${ }^{\circledR}$ verbindet eine gute Wirksamkeit mit einer schnell erreichbaren Maximaldosis: Es lassen sich sechs Maximaldosen in nur fünf Wochen erreichen. Dabei sinkt die Allergenität der Extrakte durch die patentierte Depigmentierung um bis zu $99 \%$, ohne dass
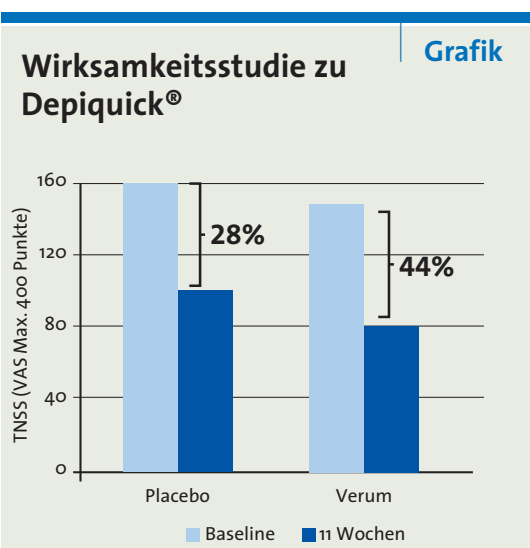

Biller $H$ et al.: Poster at EAACI, June 2009

die Immunogenität leidet. Wirksamkeit und Sicherheit der Quick-Aufdosierung mit dem depigmentierten Allergoid belegen mehrere Studien.

Brehler selbst hat prospektiv die Sicherheit einer solchen raschen Aufdosierung im Vergleich zur dreiwöchigen Aufdosie- rung mit depigmentiertem Allergoid an 303 Patienten mit Allergie gegen Baum-, Gräser-, Kräuterpollen oder Hausstaubmilben untersucht. Dabei waren systemische Reaktionen vom Grad 1 bei rascher Aufdosierung etwas häufiger (5,34 vs. 2,05\%), schwere systemische Reaktionen traten aber nicht auf, sodass Brehler das Verträglichkeitsprofil als gleich gut bewertete. Die klinische Wirksamkeit und Sicherheit der depigmentierten Allergoide konnte damit inzwischen bei etwa 1.500 Patienten dokumentiert werden. Eine aktuelle Wirksamkeitsstudie wurde im Juni 2009 auf dem europäischen Allergiekongress in Warschau vorgestellt. Depiquick ${ }^{\circledR}$ reduzierte danach bei 72 Gräserpollenallergikern mit Rhinokonjunktivitis und/oder leichtem allergischem Asthma innerhalb von elf Wochen den Gesamtwert der nasalen Symptome (TNSS) im Median signifikant stärker als Placebo.

Industriesymposium „Therapie und Abrechnung - Was gibt's Neues bei SCIT?", Berlin, 4.9.2009 (Veranstalter: Novartis Pharma).

\section{Erblich bedingter Haarverlust lässt sich bremsen}

- Etwa $30 \%$ der Patienen mit androgenetischer Alopezie (AGA) sind Frauen. Je früher die Diagnose gestellt und mit der Therapie einer AGA begonnen wird, desto erfolgversprechender sei sie, betonte Dr. Andreas Finner, Berlin, da die Verkleinerung der Haarfollikel im Rahmen der Alopecia androgenetica im Allgemeinen nicht reversibel sei.

Neben der Anamnese ist es unerlässlich, das Muster der Haardichteminderung zu erfassen und eine vernarbende Alopezie auszuschließen. Hinzu kommen die dermatoskopische Untersuchung und der Zupftest. Bei Frauen mit AGA gilt es auch, Hormonauffälligkeiten auszuschließen. Allerdings finden sich als Ursache nur selten erhöhte Androgenspiegel im Blut betroffener Frauen. Viel häufiger handelt es sich dagegen um eine erhöhte Empfindlichkeit der Haarwurzeln auf Dihydrotestosteron (DHT).

Hier besteht die Möglichkeit einer äußerlichen Behandlung mit 17 $\alpha$-Estradiol-Lösung (Pantostin ${ }^{\circledR}$ ), die einmal täglich auf die Kopfhaut aufgetragen wird und für den frühen anlagebedingten Haarausfall zugelassen ist. $17 \alpha$-Estradiol hemmt die $5 \alpha$ Reduktase, die Testosteron in DHT umwandelt. Dadurch lasse sich der fortschreitende Haarverlust wirksam stoppen, so Finner. Das habe eine offene multizentrische Studie mit 1.975 Patienten belegt. Der Haarausfall über fünf Tage, der bei Therapiebeginn noch bei 117 Haaren lag, reduzierte sich nach dreimonatiger Behandlung auf 83 und nach sechs Monaten auf 60 Haare.

Die Ärzte beurteilten die therapeutische Wirkung zu $80 \%$ mit sehr gut bis gut. Diese Bestnoten vergaben $78 \%$ der Patienten für den Behandlungserfolg. Die Vorteile der $17 \alpha$-Estradiol-Lösung sieht Finner in der einmal täglichen Anwendung und der guten Verträglichkeit. „Nach 6 bis 12 Monaten lässt sich beurteilen, ob die Therapie wirkt. Falls ja, ist das eine Dauerbehandlung“, so der Haarexperte.

bm

Pressegespräch „Therapie der Alopezie mit Pantostin ${ }^{\circledR}$ und Pantovigar ${ }^{\circledR}$ " anlässlich der Jahrestagung der EADV, Berlin, 8.10. 2009 (Veranstalter: Merz Pharmaceuticals).

\section{THEO - für mehr Compli- ance}

- Nur einer von drei bis vier Patienten bleibt auch im dritten Jahr einer SIT noch bei der Stange. THEO - der THErapie-Organizer für Allergiker - soll Patienten die Therapietreue erleichtern. Dieser AllergietherapiePass wurde speziell entwickelt, um Patienten zur Durchführung einer dreijährigen Hyposensibilisierung zu motivieren. Er besteht aus einem Organizer-Teil, in dem detailgenau die gesamte Behandlung dokumentiert wird, bietet aber darüber hinaus noch viele weitere Informationen.

THEO kann kostenlos abgefordert werden bei

Allergopharma

Joachim Ganzer KG 